\title{
Jeffrey Young: «Eigentlich waren sie schon ihr ganzes Leben lang depressiv»"
}

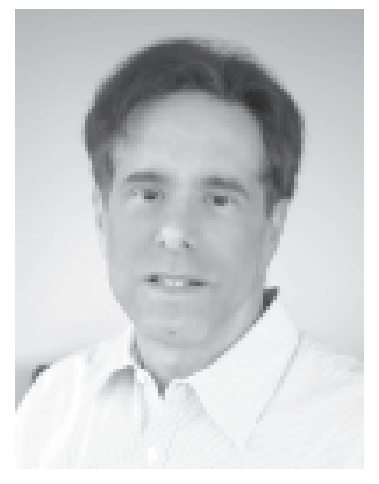

Dr. Jeffrey Young ist der Begründer der Schematherapie. Er studierte zunächst bei Joseph Wolpe Verhaltenstherapie, später arbeitete er als Direktor für Forschung und Ausbildung an der Klinik von Aaron Beck, mit dem er zahlreiche Arbeiten über Depression und Kognitive Therapie veröffentlicht hat. Heute ist er Forscher, Universitätslehrer, Ausbilder und praktizierender Schematherapeut. Er ist Direktor des Schematherapie-Institutes in New York, Manhattan. Zur Zeit schreibt Dr. Young hauptsächlich über Persönlichkeitsstörungen, insbesondere Borderline- und narzisstische Störungen, schwierige chronische Patienten und Schematherapie. Das Interview fand in Stockholm während eines Workshops über Schematherapie statt. Interviewer war Dr. Heinrich Berbalk, Professor für Psychologie an der Universität Hamburg.
Ich möchte mit Ihnen gerne über die Schematherapie sprechen. Lassen Sie uns mit der Entwicklung der Schematherapie anfangen und wie sie mit Ihrer eigenen Entwicklung zusammenhängt.

Jeffrey Young: Meine ursprüngliche Ausbildung war als Verhaltenstherapeut bei Wolpe. Ich war ein großer Anhänger der Verhaltenstherapie und habe sie mit Begeisterung angewandt. Als ich jedoch außerhalb von Wolpes Klinik mit nicht-phobischen Patienten arbeitete, erkannte ich die Grenzen seiner Verhaltenstherapie. Dabei stieß ich auf das Buch «Kognitive Therapie und emotionale Störungen» von Aaron Beck. Es schien mir reichhaltiger als Wolpes Ansatz, auch was meine eigenen Sichtweisen anging. Ich beschloss, meine Dissertation über Kognitive Therapie und Einsamkeit zu schreiben. Ich konnte zeigen, dass Kognitionen eine große Rolle für die Intensität des Einsamkeitserlebens spielen. Was dann passierte, war reiner Zufall. Ich gab ein Seminar über verschiedene Theorien zur Psychotherapie, unter anderem über kognitive Therapie. Aaron Beck war zu dieser Zeit auch in Pennsylvania. Wir trafen uns, diskutierten meine Dissertation und mein Seminar, und er bot mir eine Stelle an. Drei Jahre arbeitete ich mit ihm zusammen, zunächst als Doktorand, später als Direktor für Lehre und Forschung. Die Kognitive Therapie war bei schwer depressiven Patienten sehr effektiv. Die Erfolgsquote lag bei $75 \%$. Nachdem ich Becks Klinik verlassen hatte und mich mit einer eigenen Praxis niedergelassen hatte, ging es mir wie zuvor schon bei der Verhaltenstherapie: Viele Patienten waren komplexere Fälle als die in Becks Klinik. Sie waren schon seit Jahren in Psychotherapie, hatten Persönlichkeitsstörungen, dysthyme Störungen, generalisierte Angststörungen oder passten in keine Kategorie. Erst später erkannte ich, dass diese Patienten, die nicht auf die Therapie ansprachen, alle ein lebenslanges Muster irgendeines psychologischen Problems hatten.

Was sahen Sie als die Ursachen für diesen ausgebliebenen Erfolg?

Jeffrey Young: Ehrlich gesagt, hatte ich keine Ahnung. Ich brachte eine Gruppe von Therapeuten zusammen, die alle in Becks Institut gewesen waren und sich privat niedergelassen hatten. Wir sprachen über die Patienten, die sich nicht so gut machten, und versuchten zu erkennen, was sie gemeinsam hatten. Es wurde klar, dass bei diesen Patienten grundsätzlichere Themen am Werk waren, z.B. Bindungsängste oder massive Selbstwertprobleme, also mehr als nur eine akute Depression. Wir hatten es mit chronischen Problemen zu tun, die sehr früh begonnen hatten. Patienten, deren Probleme erst später entstanden, schienen sich besser zu entwickeln als sol-

\begin{tabular}{ll}
\hline KARGER & @ 2003 S. Karger GmbH, Freiburg \\
Fax +497614520714 & Accessible online at: \\
$\begin{array}{l}\text { E-mail Information@Karger.de } \\
\text { www.karger.com }\end{array}$ & www.karger.com/ver
\end{tabular}

Prof. Dr. Heinrich Berbalk

Psychologisches Institut III, Universität Hamburg

Von-Melle-Park 5, D-20146 Hamburg

Tel. +49 171 483-9090, Fax -9091

E-mail berbalk@uni-hamburg.de 
che, die ihr Problem schon immer hatten, die «eigentlich schon ihr ganzes Leben lang» depressiv waren. Die Erfolgsrate bei diesen Patienten war schlecht. Daher wollte ich herausfinden, was ich der Kognitiven Therapie hinzufügen müsste, um diesen Patienten gerecht zu werden.

In meiner Beobachtung hat sich die Schematherapie im Laufe der Zeit zu einem eigenen Ansatz entwickelt. Diese Sichtweise teile ich mit David Barlow und Paul Wachtel, der ja für Psychotherapieintegration bekannt ist.

Jeffrey Young: Zunächst nahm ich Techniken aus der Gestalttherapie hinzu. Obwohl die Kognitive Therapie meiner eigenen Denkweise sehr entsprach, halfen mir doch imaginative Techniken zu den tieferen Schemata vorzudringen. Ich erweiterte das Behandlungsmodell und begann, diese Schemata der Patienten zu katalogisieren. Ich benutzte den Begriff «Schema», wie Beck seinerzeit auch. Ich fand «Schema» aussagekräftiger als «Grundannahme». Obwohl Kognitive Therapeuten damals bereits mit Grundannahmen arbeiteten, erschien mir dies noch zu oberflächlich. Grundannahmen waren Aussagen wie: «Wenn ich mich sehr anstrenge, werde ich ein guter Mensch sein.» Damit konnte man Erfahrungen wie «Vernachlässigung erleben», «sich unterwerfen» oder «Probleme mit Selbst- und Fremdbestimmung» nicht einfangen. Warum entwickelt jemand z.B. das Schema, «dass ihn niemand lieben kann»? In einer Gruppe Kognitiver Psychotherapeuten erweiterten wir das Modell kontinuierlich und suchten für jedes neue Schema eine neue Erklärung. Irgendwann sagten Kollegen, das ist keine Modellerweiterung mehr, sondern ein ganz neues Modell. Obwohl es nicht meine Absicht gewesen war, kam der Zeitpunkt, wo die kognitiven Techniken eben nur noch gleichwertig neben den anderen standen. Verhaltens- und Erlebenskomponenten, die therapeutische Beziehung und die kognitiven Elemente waren gleichermaßen wichtig und es war nicht mehr angemessen, nur von Kognitiver Therapie zu sprechen.

$\mathrm{Zu}$ dem neuen Modell gehörte auch die Erweiterung um die Entwicklungsthemen - wie sind die Schemata entstanden? - , die in der Kognitiven Therapie immer noch fehlen. Die Arbeit mit chronischen Patienten ist sehr schwierig, wenn man nicht erklären kann, wie die Störung entstanden ist. Ich stellte fest, dass die Themen oder Schemata auch bei Menschen, die nicht in Therapie waren, die gleichen waren. Die Schemata entwickeln sich aus universellen Bedürfnissen. Das war die interessante Entdeckung. Alle Menschen kämpfen z.B. in irgendeiner Form mit Verlassensängsten; die Patienten sind nicht sehr anders, nur oft extremer. $\mathrm{Zu}$ diesem Zeitpunkt begann ich, den Schemaansatz als eine generelle Art anzusehen, wie man Menschen betrachten und ihre persönliche Entwicklung verstehen kann.
Es ist also wichtig, die Schemata zu erkennen und ihre Entstehung zu verstehen. Sie betonen aber, dass das nicht reicht. Es ist auch wichtig zu wissen, wie man mit den Schemata umgeht. Warum ist dies wichtig?

Jeffrey Young: Ich kam mit bestimmten Patienten nicht weiter. Bat ich sie, sich ein Bild von sich als Kind vorzustellen, sahen sie nichts oder wollten nicht darüber nachdenken oder sprechen. Sie hatten Mechanismen entwickelt, um bestimmte Gefühle nicht zu haben oder um kein Bewusstsein für die schmerzhaften Erlebnisse zu haben.

Ich begann mit Patienten, die die Kindheitsbetrachtung als Zeitverschwendung abtaten. Ich vermutete, sie wollten nicht darüber nachdenken, weil es schmerzhaft war. So entwickelte ich die Vorstellung von Schemabewältigungsstilen. Da die Patienten offensichtlich nicht nur Schemata entwickelt hatten, sondern auch Strategien, um diese zu umgehen, sprach ich von Bewältigungsstilen. Schwierige Patienten hatten nicht nur starke Schemata, sondern auch ausgefeilte Bewältigungsmechanismen entwickelt. Um überhaupt Zugang zu den Schemata zu bekommen, musste ich zunächst die Bewältigungsmechanismen überwinden und den Patienten helfen zu verstehen, wie diese funktionieren. Die Patienten brachen dabei aber nicht etwa zusammen. Im Gegenteil. Sie waren ganz aufgeregt, endlich herauszufinden, was hinter ihren Problemen steckte. Sie waren erleichtert, denn aufgrund ihrer Schemabewältigungsmechanismen hatten sie nie eine Ahnung gehabt, warum sie solche Probleme hatten.

Zu den Schemata, der Schemaentwicklung und der Schemabewältigung kommt als jüngste Entwicklung der Schematherapie das Konzept der Modi hinzu. Wie kam es zu dieser Erweiterung?

Jeffrey Young: Es begann mit zwei Borderline-Patientinnen, bei denen ich mit meinen üblichen Strategien zum Umgang mit Schemata nicht weiterkam. Beide zeigten starke Ausprägungen bei fast allen der von uns gefundenen früh erworbenen hinderlichen Schemata. Außerdem wechselten ihre Launen von einem Moment zum nächsten. Jeder, der mit Borderline-Patienten gearbeitet hat, kennt das. Einen Augenblick sind sie wütend und finden einen furchtbar, im nächsten Moment lieben sie dich und wollen von dir adoptiert werden, dann wieder fühlen sie sich furchtbar und wollen sich schneiden, um sich zu bestrafen oder zu beruhigen. Ich erkannte, dass es im Grunde genommen verschiedene Teile dieser Person waren, die zu bestimmten Zeiten in den Vordergrund traten. Ich wusste nicht, wie ich damit umgehen sollte, und hatte in meiner Theorie auch keine Erklärung dafür. Dann kam mir die Idee der Modi. Ein Modus hat Ähnlichkeit mit einem Gemütszustand. Bei einem Moduswechsel ändert sich die ganze Art zu funktionieren. Also nahm ich das Wort «Schemamodus». Wir fanden bei fast allen Borderline-Patienten vier konkurrierende Modi, die wir nach ihrem Entstehungskontext be- 
nannten: «Das verlassene Kind», «das wütende Kind», «der strafende Elternteil» und «der ungerührte Beschützer», der sich emotional abschirmt. Da Patienten, die sich in einem Modus befinden, keinen Zugang zu den anderen Teilen ihrer Person haben, war die Konsequenz, eine feste Struktur innerhalb der Therapie aufzugeben. Ist der Patient wütend, können wir ihn nicht so behandeln, wie wenn er deprimiert ist. Also passten wir die Therapiestrategie dem momentanen Modus an. Zunehmend wurde das Konzept auch bei narzisstischen Patienten und nicht so schweren Fällen eingesetzt und war sehr hilfreich. Seither kombinieren wir das Schemamodell mit dem Modusmodell.

In Ihren Workshops und Ihren Büchern kann man auch etwas über die Schemata und Modi der Therapeuten lernen.

Jeffrey Young: Wir haben alle Probleme. Auch für den Therapeuten ist es gut, ein Erklärungsmodell für seine Probleme zu haben. Außerdem können die Schemata des Therapeuten die Dynamik zwischen Therapeut und Patient erklären. Nehmen wir an, ein Borderline-Patient befindet sich im Modus «Wütendes Kind» und greift den Therapeuten an, indem er sagt, «du hast dich nie um mich gekümmert». Hat nun der Therapeut ein Unvollkommenheits- oder Versagensschema, das durch den Angriff aktiviert wird, könnte seine erste Reaktion sein: «Ich bin nicht gut genug»; darauf hin setzt sein Bewältigungsmechanismus ein, und er verteidigt sich. Wenn er sich verteidigt, lehnt er aber die Erfahrung des Patienten ab. Das ist für den Borderline-Patienten aber nicht sehr hilfreich. Viele Therapeuten haben Schwierigkeiten mit Narzissten zu arbeiten, da sie ihnen ständig das Gefühl geben, unfähig zu sein.

Dies zu wissen, ist auch ein Weg, um sich in einer schwierigen therapeutischen Beziehung zu distanzieren.

Jeffrey Young: Genau. Wenn du dich nicht genügend distanzieren kannst und nicht objektiv sehen kannst, was der Patient macht, passieren zwei Dinge. Zum einen kannst du dem Patienten nicht helfen, da du zu sehr damit beschäftigt bist, dich zu rechtfertigen oder über deine eigenen Fehler nachzudenken; zum anderen achtest du nicht darauf, was du selbst tust und lässt es am Patienten aus.

Der Therapeut kann aber auch dieselben Schemata haben wie der Patient und so mit ihm fühlen, dass er nicht mehr weiß, was an dessen Verhalten falsch sein soll, da er selbst genauso gehandelt hätte. Wenn man dieselben Schemata hat wie sein Patient, ist es sehr schwer, objektiv zu bleiben und Lösungen $\mathrm{zu}$ finden. Ein Therapeut, der mit seinen eigenen Modi und Schemata nicht umgehen kann, steht unter einem enormen inneren Druck, da er sich ständig Vorwürfe macht oder sich vor seinen Patienten schützen und hierfür so viel Energie aufbringen muss, dass er keine Kraft mehr für sie hat. Er verliert seinen Enthusiasmus, seine Zuversicht und brennt aus.
Eine weitere Säule in der Schematherapie ist die besondere therapeutische Beziehung, speziell die so genannte begrenzte und ausgleichende elterliche Fürsorge.

Jeffrey Young: Ich nenne es eingeschränkte elterliche Fürsorge, da ein Therapeut natürlich nicht alle Funktionen eines Elternteils erfüllen kann. Man kann den Patienten aber durch die therapeutische Beziehung mit Erfahrungen bereichern, die ihm in seiner Kindheit gefehlt haben und Ursache seiner Schemata sind. Hatte ein Patient beispielsweise protektive Eltern, ermutige ich ihn, sich seine eigenen Gedanken zu machen. Ein Patient mit einem Schema emotionaler Deprivation, der stark distanzierte Eltern hatte, erhält von mir hingegen Anweisungen und Vorschläge, ungefähr so, wie Eltern das machen würden. Was für den einen in der therapeutischen Beziehung richtig ist, kann für den anderen falsch sein. Ein Narzisst braucht klare Grenzen, ein Patient mit einem Unterwerfungsschema keinesfalls. Je mehr der Therapeut über die Schemata seines Patienten weiß, desto besser kann er seine Therapie nach den Bedürfnissen des Patienten ausrichten.

Dazu kommt, je schwerer die Störung des Patienten ist, desto wichtiger ist die Elternrolle. Bei einem Borderline-Patienten, wo wir «Vernachlässigung», «Misshandlung» und alle nur denkbaren Schemata finden, wird man eine echte Elternfigur, denn diese Patienten hatten nie wirklich Eltern.

\section{Könnten Sie uns die Unterschiede zwischen Schematherapie und Psychoanalyse erläutern?}

Jeffrey Young: Viele Psychoanalytiker würden vielleicht sagen, dass die Therapieformen sehr ähnlich sind. Viele Kognitive Therapeuten sehen es ähnlich. Da die Schematherapie sehr integrativ ist, hat sie tatsächlich Ähnlichkeiten mit allen Therapieformen. Die «Kernkonflikte» in der Psychoanalyse ähneln den Schemata, die «Abwehrmechanismen» ähneln den Schemabewältigungsstilen, und manche sagen, dass die «IchZustände» den Modi ähneln. Die Schematherapie wurzelt aber nicht in der Psychoanalyse. Ihre Konzepte sind ganz anders entwickelt worden. Der wichtigste Unterschied ist der Stil. Die meisten Analytiker lernen, dass sie neutral sein müssen. Sie beschreiten eher einen passiven Weg. Schematherapeuten zeigen persönliche Beteiligung und sind sehr aktiv. Ein anderer großer Unterschied ist das Konzept des ausgleichenden elterlichen Umsorgens als herausragendes Element der therapeutischen Beziehung in der Schematherapie. Somit unterscheidet sich die Schematherapie, trotz vorhandener theoretischen Ähnlichkeiten zur Psychoanalyse, in ihrer Durchführung und besonders im Hinblick auf die therapeutische Beziehung, von den meisten psychoanalytischen Schulen sehr. 
Sie sagten, dass der Therapeut in der Beziehung als Rollenmodell fungiert für den Modus des gesunden Erwachsenen. Dazu muss er eine echte Beziehung zu dem Patienten haben.

Jeffrey Young: Wenn die Therapie gut läuft, verinnerlicht der Patient den Therapeuten als Teil von sich. Häufig erzählen Patienten, dass sie bei bestimmten Gedanken oder Ereignissen meine Stimme im Ohr haben, die ihnen sagt, dass sie nicht aufgeben sollen oder dass sie ein guter Mensch sind, oder sie fühlen, dass sie mir wichtig sind. Manchmal gebe ich meinen Patienten kleine Kärtchen, auf denen rationale Antworten auf ihre negativen Gedanken und Gefühle stehen, und sie sagen, es ist nicht nur so, als ob sie die Antworten in ihrer Tasche haben, es ist, als ob sie mich in ihrer Tasche herumtragen. Wenn man in eine echte Beziehung mit ihnen tritt und sich mit ihnen austauscht, ermöglicht man ihnen, dass sie einen verinnerlichen und als echten Elternteil empfinden.

Vielen Dank, dass Sie sich die Zeit für das Gespräch genommen haben. Ich möchte Ihnen auch noch zu Ihrem neuen Buch «Schema Therapy. A Practitioner's Guide» gratulieren, dem ich eine weite und rasche Verbreitung wünsche. 